\title{
HOMOLOGY OF SMOOTH SPLINES: GENERIC TRIANGULATIONS AND A CONJECTURE OF STRANG
}

\author{
LOUIS J. BILLERA
}

Dedicated to the memory of Joseph J. Billera

\begin{abstract}
For $\Delta$ a triangulated $d$-dimensional region in $\mathbf{R}^{d}$, let $S_{m}^{r}(\Delta)$ denote the vector space of all $C^{r}$ functions $F$ on $\Delta$ that, restricted to any simplex in $\Delta$, are given by polynomials of degree at most $m$. We consider the problem of computing the dimension of such spaces. We develop a homological approach to this problem and apply it specifically to the case of triangulated manifolds $\Delta$ in the plane, getting lower bounds on the dimension of $S_{m}^{r}(\Delta)$ for all $r$. For $r=1$, we prove a conjecture of Strang concerning the generic dimension of the space of $C^{1}$ splines over a triangulated manifold in $\mathbf{R}^{2}$. Finally, we consider the space of continuous piecewise linear functions over nonsimplicial decompositions of a plane region.
\end{abstract}

1. Introduction. Let $\Delta$ be a finite $d$-dimensional simplicial complex [16, p. 7] (rectilinearly) embedded in $\mathbf{R}^{d}$, i.e., we think of $\Delta$ as giving a triangulation of a compact region in $\mathbf{R}^{d}$ in such a way that each simplex in $\Delta$ is the convex hull of its vertices. We will often just denote the region itself by $\Delta$; for example, we write $\Delta \subseteq \mathbf{R}^{d}$. The exact nature of the embedding will be an issue later on. We assume throughout that $\Delta$ is pure, that is, each maximal simplex has dimension $d$. We will also assume, without loss of generality, that $\Delta$ is connected.

For nonnegative integers $m$ and $r$, we define $S_{m}^{r}(\Delta)$ to be the set of all piecewise polynomial functions on $\Delta$ which are of degree at most $m$ and are smooth of order $r$, i.e., all functions $F: \Delta \rightarrow \mathbf{R}$ such that

(1.1) $\left.F\right|_{\sigma}$ is a (real) polynomial of degree $\leq m$, for each $\sigma \in \Delta$ and

(1.2) $F$ is continuously differentiable of order $r$.

Such functions are often called splines or finite elements. The set $S_{m}^{r}(\Delta)$ forms a vector space over $\mathbf{R}$; it is the aim of this paper to study its dimension.

This problem was first formally introduced by Strang $[\mathbf{2 3}, \mathbf{2 4}]$, who traces its history to a paper of Courant [12], where the idea of approximation by continuous piecewise polynomial (specifically, piecewise linear) functions is suggested. In the case of continuous piecewise linear functions (i.e., $r=0, m=1$ ), it is straightforward to see that the dimension is $f_{0}$, the number of vertices of $\Delta$; in particular, a basis for $S_{1}^{0}(\Delta)$ is given by those piecewise linear functions having value 1 on some vertex and 0 on all others. For a detailed discussion of all the spaces $S_{m}^{0}(\Delta)$, see

Received by the editors August 27, 1987.

1980 Mathematics Subject Classification (1985 Revision). Primary 41A15, 65D07; Secondary 05A15, 52A37, 55N25, 57Q15.

Supported, in part, by National Science Foundation Grants DMS-8403225 and DMS-8703370. 
[8], where, for example, it is shown that $\operatorname{dim} S_{m}^{0}(\Delta)=\sum_{j=0}^{d} f_{j}\left(\begin{array}{c}m-1 \\ j\end{array}\right)$ for $m>0$, where $f_{j}$ is the number of $j$-dimensional faces of $\Delta$.

We are interested here in developing methods to deal with the spaces $S_{m}^{r}(\Delta)$ for $r>0$. We use these methods, in the case $d=2$ and $r=1$, to verify a conjecture of Strang $[\mathbf{2 3}]$ when $\Delta$ is a 2-manifold.

The case $d=2$ of planar triangulations has received considerable attention since Strang's 1973 paper [23] (see also [24]). Here, a heuristic calculation was suggested to compute the dimensions of the spaces $S_{m}^{1}(\Delta)$, leading to a conjecture, which we state as follows.

STRANG'S CONJECTURE. For a generic embedding of a planar 2-manifold $\Delta$,

$$
\operatorname{dim} S_{m}^{1}(\Delta)=\left(\begin{array}{c}
m+2 \\
2
\end{array}\right) f_{2}-(2 m+1) f_{1}^{\circ}+3 f_{0}^{\circ},
$$

where $f_{2}$ is the number of triangles in $\Delta, f_{1}^{\circ}$ and $f_{0}^{\circ}$ the number of interior edges and vertices, respectively.

To be specific, let $\Delta$ be a simplicial complex with $n$ vertices. We say a property of $\Delta$ holds for generic embeddings of $\Delta$ in the plane, or holds generically, if there is a nonzero real polynomial $p$ of $2 n$ variables such that the property is true for any embedding of $\Delta$ in $\mathbf{R}^{2}$, with vertex locations $\left(x_{i}, y_{i}\right), i=1, \ldots, n$, such that $p\left(x_{1}, y_{1}, \ldots, x_{n}, y_{n}\right) \neq 0$.

While generic embeddings were not explicitly mentioned by Strang, he was already aware of the fact that the dimension of the spline spaces might jump in some special positions. In particular he noted that the number of smooth quadratics on a rectangle triangulated by its two crossing diagonals was higher than the case in which the interior edges did not lie on two straight lines.

The first published results on this problem were by Morgan and Scott [17], who proved the conjecture for $m \geq 5$ by showing that for any embedding of $\Delta$, the dimension of $S_{m}^{1}(\Delta)$ is given by (1.3) plus the number of rectangles triangulated by crossing diagonals. Their proof gave an explicit basis for $S_{m}^{1}(\Delta), m \geq 5$. In an unpublished paper written around the same time [18], they gave an example which showed that their result was false for $m=2$. (See also [19] or [10].) Later, Schumaker [19] showed that the Morgan-Scott dimension was a lower bound for all $m \geq 2$ and gave a similar lower bound for the dimension of the spaces $S_{m}^{r}(\Delta)$ when $m \geq r+1$. (When $m \leq r$, there are only global polynomials.)

Since then much work has been done on special triangulations, usually rectangular grids with diagonals (e.g., [9] and [10]), or on special subspaces of splines (e.g., $B$-splines). For a survey of this work, especially the latter approach, see [14]. In [19], upper bounds are derived for general planar triangulations, while in [22], simplices defined by more general hypersurfaces are considered.

In the past few years, there has been much progress on this problem [1-6]. Alfeld and Schumaker [6] extended the Morgan-Scott result for all $r$ by showing that Schumaker's lower bound is in fact the dimension of $S_{m}^{r}(\Delta)$ when $m \geq 4 r+1$. Along with Piper $[\mathbf{4}, \mathbf{5}]$, they constructed explicit bases for these spaces. Alfeld, Piper and Schumaker [3] also extended the Morgan-Scott $C^{1}$ results to $m=4$.

The purpose of this paper is to develop some algebraic machinery to study the spaces of smooth piecewise polynomial functions on triangulations of any dimension. 
In $\S 2$, an algebraic criterion is given for local smoothness of piecewise polynomial functions on fairly general pseudomanifolds. The basic idea of this criterion can be found in the early paper of Wang [25] and the lecture notes of Ciarlet [11]. $\S 3$ gives a homological approach to piece together these local conditions. This is specialized to the case of $d=2$ in $\S 4$ and used to produce some easy lower bounds on the dimension of $S_{m}^{r}(\Delta)$ for all $m \geq r+1$. The essential elements in the proof of Strang's conjecture are also developed in this section. In $\S 5$, the proof of Strang's conjecture is completed, making use of a result of Whiteley [28]. $\S 6$ discusses possible extensions, including the study of piecewise linear functions on nonsimplicial subdivisions of planar regions and splines with boundary constraints.

Given the results of Morgan and Scott and of Alfeld, Piper and Schumaker, the essential contribution of this work to Strang's conjecture is in the case of degrees $m=2$ and $m=3$. It is here (at least for $m=2$ ) that a global notion of genericity of triangulations comes into play.

While it is likely that the essential elements of the proof of Strang's conjecture given here can be "simplified" to exclude any mention of homology (most likely with longer arguments; after all, it is just linear algebra), we feel that the importance of homological methods is that they provide a unified approach to many problems of this sort. It basically gives a way of doing a lot of complicated linear algebra in a very organized way and very possibly could be of use in other problems in this area.

Much of the original interest in the study of smooth piecewise polynomial functions was due to their application in the solution of partial differential equations by the finite element method. Recently, they have been attracting much interest because of new applications in the field of computational geometry. These have involved mostly questions in dimension 2 , concerning piecewise polynomial approximation of surfaces for purposes of automated design and control. (See, for example, [7] or the references in [14].)

This work has benefited from conversations with Marshall Cohen, John Hubbard, Gil Kalai, Alex Rosenberg and Walter Whiteley. In particular, Whiteley's solution of the spline matrix problem [28] is essential to the main result here.

2. Some algebraic preliminaries. We first develop an algebraic condition that is both necessary and sufficient for a piecewise polynomial function on a (sufficiently connected) $d$-dimensional complex $\Delta$ to be smooth of order $r$. The necessity of this condition is discussed in [11] and [25] (see also [9]) for the case $d=2$. The former suggests a proof that can be used for general $d$; we show here that the basic idea of the latter also extends.

LEMMA 2.1. Let $l$ be an affine polynomial (i.e., of degree at most 1) and $f$ be an arbitrary polynomial in $k\left[x_{1}, \ldots, x_{d}\right]$, where $k$ is any infinite field. Suppose $f$ vanishes at any point in $k^{n}$ at which $l$ vanishes. Then $l \mid f$ in $k\left[x_{1}, \ldots, x_{d}\right]$.

Proof. Let $R=k\left[x_{2}, \ldots, x_{d}\right]$, so $k\left[x_{1}, \ldots, x_{d}\right]=R\left[x_{1}\right]$. We can assume $l=$ $x_{1}+a_{2} x_{2}+\cdots+a_{d} x_{d}+a_{0}$, and define $a=-\left(a_{2} x_{2}+\cdots+a_{d} x_{d}+a_{0}\right) \in R$. Considering $f=f\left(x_{1}\right)$ as an element of $R\left[x_{1}\right]$, we see that $f(a)=f\left(-a_{2} x_{2}-\cdots-a_{d} x_{d}-a_{0}\right)=0$ for all values of $x_{2}, \ldots, x_{d} \in k$, by hypothesis. Thus $f(a)=0$ in $R$. By [30, Corollary 1, p. 31], $\left(x_{1}-a\right) \mid f\left(x_{1}\right)$ in $R\left[x_{1}\right]$ and so $l \mid f$ in $k\left[x_{1}, \ldots, x_{d}\right]$. 
In [9], this result is proved for $d=2$ for any irreducible $l$ (not necessarily affine) by means of Bezout's theorem on plane curves. That proof does not generalize to $d>2$. In [25], however, there is another proof given for the affine case in $d=2$ that is basically the same as the one above. Using Lemma 2.1, the following result is proved exactly as in [9, Lemma 2.7].

LEMMA 2.2. Suppose $F \in S_{m}^{r}(\Delta)$ for some $d$-complex $\Delta \subset \mathbf{R}^{d}$ and $r \geq 0$. Suppose $\sigma_{1}, \sigma_{2} \in \Delta$ are two d-simplices such that $\tau=\sigma_{1} \cap \sigma_{2}$ has dimension $d-1$. Then if $l$ is a nontrivial affine form which vanishes on $\tau$, we have

$$
l^{r+1} \mid\left(p_{1}-p_{2}\right)
$$

where $p_{i}=\left.F\right|_{\sigma_{i}}, i=1,2$.

We say a $d$-complex $\Delta$ is strongly connected if for any two $d$-simplices $\sigma, \sigma^{\prime} \in \Delta$, there is a sequence of $d$-simplices

$$
\sigma=\sigma_{1}, \sigma_{2}, \ldots, \sigma_{k}=\sigma^{\prime}
$$

such that for each $i<k, \sigma_{i} \cap \sigma_{i+1}$ has dimension $d-1$. Here we say that $\sigma_{i}$ and $\sigma_{i+1}$ are adjacent. For $\tau \in \Delta$, define the link of $\tau, \operatorname{lk}(\tau)=\{\sigma \in \Delta \mid \sigma \cap \tau=\varnothing$, $\sigma \cup \tau \in \Delta\}$. (For the purposes of this definition, we are considering a simplex to be merely the set consisting of its vertices.)

We note here that it follows from Lemma 2.2 that if $\Delta$ is a strongly connected $d$ complex and $r \geq m$, then $S_{m}^{r}(\Delta)$ is exactly the space of global polynomial functions of degree at most $m$, i.e., functions $F$ satisfying (1.1) such that $\left.F\right|_{\sigma}=\left.F\right|_{\sigma^{\prime}}$ for all $\sigma, \sigma^{\prime} \in \Delta$.

THEOREM 2.4. Suppose $\Delta$ is a strongly connected $d$-complex such that all links of simplices are also strongly connected complexes. Let $F$ be a piecewise polynomial function satisfying (1.1) and $r \geq 0$. Then $F \in S_{m}^{r}(\Delta)$ if and only if (2.3) holds for each pair $\sigma_{1}, \sigma_{2}$ of adjacent d-simplices in $\Delta$.

Proof. Necessity is Lemma 2.2. To see sufficiency, note first that (2.3) implies that $F$ is an $r$-fold smooth function on any pair of adjacent simplices $\sigma_{1}$ and $\sigma_{2}$; the only problem could be on $\tau=\sigma_{1} \cap \sigma_{2}$, but (2.3) shows there is no problem here either. More generally, let $x$ be any point in $\Delta$ (as a subset of $\mathbf{R}^{d}$ ) and let $\tau$ be a minimal simplex of $\Delta$ containing $x$. That $F$ has continuous $r$ th order partial derivatives at $x$ follows from the observation above and the fact that $\operatorname{lk}(\tau)$ is strongly connected.

We note here that if $\Delta$ is a triangulation of a connected $d$-manifold, then $\Delta$ satisfies the conditions of the theorem $[\mathbf{1 6}, \S 63]$. In fact, given that $\Delta$ is embedded in $\mathbf{R}^{d}$, the hypothesis is equivalent to asking that $\Delta$ and all its links are pseudomanifolds [16].

Let $P_{m}$ denote the $\mathbf{R}$-subspace of the polynomial $\operatorname{ring} A=\mathbf{R}\left[x_{1}, \ldots, x_{d}\right]$ consisting of all polynomials of degree at most $m$. This subspace has dimension $\left(\begin{array}{c}m+d \\ d\end{array}\right)$. We wish to consider the dimension of certain quotients of $P_{m}$.

Let $\tau$ be a simplex in $\mathbf{R}^{d}$, i.e., the convex hull of affinely independent points. Let

$$
I_{\tau}=\left\{f \in A|f|_{\tau}=0\right\}
$$

$I_{\tau}$ is the ideal in $A$ of all polynomials vanishing on aff $(\tau)$, the affine hull of $\tau$. In fact, we have more. 
Proposition 2.5. If $0 \leq \operatorname{dim} \tau \leq d-1$, then

$$
I_{\tau}=\left\langle f \in A|f|_{\mathrm{aff}(\tau)}=0, \operatorname{deg} f=1\right\rangle,
$$

where $\left\langle f_{\alpha}\right\rangle$ denotes the ideal spanned by the $f_{\alpha}$.

ProOF. First, suppose aff $(\tau)=\left\{x \in \mathbf{R}^{d} \mid x_{j}=0, j \leq k\right\}$, where $k=d-\operatorname{dim} \tau$. Then if $f \in I_{\tau}$, the restriction of $f$ to aff $(\tau)$ gives a polynomial in $\mathbf{R}\left[x_{k+1}, \ldots, x_{d}\right]$ which vanishes on an open set in $\mathbf{R}^{d-k}$; thus it must vanish on all of $\mathbf{R}^{d-k}=\operatorname{aff}(\tau)$. So $I_{\tau}$ is the kernel of the $\mathbf{R}$-algebra map

$$
\mathbf{R}\left[x_{1}, \ldots, x_{d}\right] \rightarrow \mathbf{R}\left[x_{k+1}, \ldots, x_{d}\right]
$$

that sends $x_{j} \rightarrow 0$ for $j \leq k$ and $x_{j} \rightarrow x_{j}$ for $j>k$. This is $\left\langle x_{1}, \ldots, x_{k}\right\rangle$, which is easily seen to be the desired ideal.

In the general case, one can, by an invertible affine transformation of $\mathbf{R}^{d}$, transform aff $(\tau)$ into a set of the form considered above. Suppose, under this transformation, $x_{j} \rightarrow y_{j}$ where each $y_{j}$ is of the form $a_{0}+a_{1} x_{1}+\cdots+a_{d} x_{d}$. Then $y_{1}, \ldots, y_{d}$ also generate $A$ as a polynomial ring, and the proof follows from the first case.

In what follows, we adopt the convention that $\left(\begin{array}{l}a \\ b\end{array}\right)=0$ if $a<b$, except that $\left(\begin{array}{c}-1 \\ 0\end{array}\right)=1$.

PROPOSITION 2.6. For $m \geq r \geq 0$,

$$
\operatorname{dim} P_{m} /\left(P_{m} \cap I_{\tau}^{r+1}\right)=\sum_{j=0}^{r}\left(\begin{array}{c}
m+i-j \\
i
\end{array}\right)\left(\begin{array}{c}
j+d-i-1 \\
j
\end{array}\right),
$$

where $i=\operatorname{dim} \tau$.

PROOF. In the case $i=d, I_{\tau}=0$, and both sides of the equation yield $\left(\begin{array}{c}m+d \\ d\end{array}\right)$. Otherwise, we can assume, as in the proof of the last proposition, that aff $(\tau)=\{x \in$ $\left.\mathbf{R}^{d} \mid x_{j}=0, j \leq d-i\right\}$ and so $I_{\tau}=\left\langle x_{1}, \ldots, x_{d-i}\right\rangle$. In this case $P_{m} \cap I_{\tau}^{r+1}$ is spanned by all monomials of degree at most $m$ in $x_{1}, \ldots, x_{d}$ that, when restricted to the variables $x_{1}, \ldots, x_{d-i}$, yield monomials of degree at least $r+1$. So $P_{m} /\left(P_{m} \cap I_{\tau}^{r+1}\right)$ is spanned by all monomials (of degree at most $m$ ) which can be formed by the product of a monomial of degree $j$ in $x_{1}, \ldots, x_{d-i}, 0 \leq j \leq r$, with a monomial of degree at most $m-j$ in the remaining $i$ variables. There are $\left(\begin{array}{c}j+d-i \\ j\end{array}\right)$ of the former and $\left(\begin{array}{c}m+i-j \\ i\end{array}\right)$ of the latter.

Note that if $r \geq m$, then $P_{m} /\left(P_{m} \cap I_{\tau}^{r+1}\right)=P_{m}$, which has dimension $\left(\begin{array}{c}m+d \\ d\end{array}\right)$.

Finally, let us note that if $\tau, \sigma \in \Delta$ are such that $\tau \subset \sigma$, then $I_{\sigma} \subset I_{\tau}$. Thus any R-linear map $\bar{\varphi}: P_{m} \rightarrow P_{m}$ such that $\bar{\varphi}\left(P_{m} \cap I_{\sigma}^{r+1}\right) \subset P_{m} \cap I_{\tau}^{r+1}$ induces a unique map $\varphi: P_{m} /\left(P_{m} \cap I_{\sigma}^{r+1}\right) \rightarrow P_{m} /\left(P_{m} \cap I_{\tau}^{r+1}\right)$ such that the square,

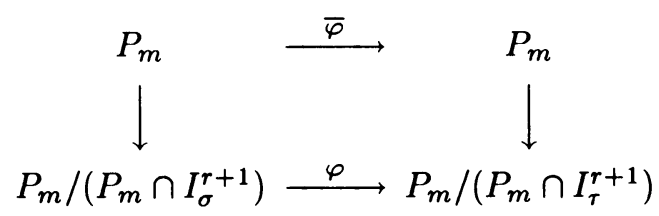

with the downward maps being the canonical projections, commutes. 
3. Homology theory for $S_{m}^{r}(\Delta)$. For the (pure) $d$-complex $\Delta$, let $\Delta_{i}$ be the set of $i$-dimensional faces ( $i$-faces) of $\Delta$ and $\Delta_{i}^{\circ}$ the set of $i$-faces in the interior of $\Delta$. If $\Delta$ has the property that each $(d-1)$-face is in one or two $d$-faces (e.g., if $\Delta$ is a pseudomanifold), then $\Delta_{i}^{\circ}=\Delta_{i} \backslash(\partial \Delta)_{i}$, where $\partial \Delta$ is the complex consisting of all those $(d-1)$-faces lying on just one $d$-face (as well as all subsets of these).

Consider the complex of $\mathbf{R}$ vector spaces, for $m \geq 0$ and $r \geq 0$,

$$
\begin{array}{r}
\mathscr{C}^{r}: 0 \rightarrow \bigoplus_{\sigma \in \Delta_{d}} P_{m} \stackrel{\partial_{d}}{\longrightarrow} \bigoplus_{\tau \in \Delta_{d-1}^{\circ}} P_{m} /\left(P_{m} \cap I_{\tau}^{r+1}\right) \stackrel{\partial_{d-1}}{\longrightarrow} \bigoplus_{\lambda \in \Delta_{d-2}^{\circ}} P_{m} /\left(P_{m} \cap I_{\lambda}^{r+1}\right) \\
\rightarrow \cdots \stackrel{\partial_{1}}{\longrightarrow} \bigoplus_{v \in \Delta_{0}^{\circ}} P_{m} /\left(P_{m} \cap I_{v}^{r+1}\right) \rightarrow 0 .
\end{array}
$$

Here, the maps $\partial_{i}$ are induced by the usual simplicial boundary maps $\bar{\partial}_{i}$ used to compute the relative homology $H_{*}\left(\Delta, \partial \Delta ; P_{m}\right)$ of the pair $(\Delta, \partial \Delta)$ with coefficients in the additive group $P_{m}$. We choose the orientation of $\Delta$ so that whenever $\sigma_{1}, \sigma_{2} \in$ $\Delta_{d}$ and $\tau=\sigma_{1} \cap \sigma_{2} \in \Delta_{d-1}^{\circ}$ then the coefficient of $\tau$ in $\bar{\partial}_{d}\left(\sigma_{1}\right)$ is \pm 1 while in $\bar{\partial}_{d}\left(\sigma_{2}\right)$ it is $\mp 1$. (One way to accomplish this is to order all the vertices of $\Delta, v_{1}, v_{2}, \ldots, v_{n}$. If $\sigma=\left\{v_{i_{0}}, v_{i_{1}}, \ldots, v_{i_{d}}\right\}, i_{0}<i_{1}<\cdots<i_{d}$, and $\tau=\sigma \backslash\left\{v_{i_{j}}\right\}$, then choosing the coefficient of $\tau$ in $\bar{\partial}_{d}(\sigma)$ to be $(-1)^{j} \operatorname{sign}\left[\operatorname{det}\left(v_{i_{1}}-v_{i_{0}}, \ldots, v_{i_{d}}-v_{i_{0}}\right)\right]$ yields the desired property.)

The homology $H_{*}\left(\mathscr{C}_{m}^{r}\right)$ of the complex $\mathscr{C}_{m}^{r}$ is of interest in studying $C^{r}$ piecewise polynomials of degree at most $m$. In particular, we have the following.

THEOREM 3.2. Let $\Delta$ be a strongly connected $d$-complex with strongly connected links. Then for $m \geq 0$ and $r \geq 0$,

$$
S_{m}^{r}(\Delta)=H_{d}\left(\mathscr{C}_{m}^{r}\right)
$$

PROOF. Since $H_{d}\left(\mathscr{C}_{m}^{r}\right)=\operatorname{ker}\left(\partial_{d}\right)$, the result follows from Theorem 2.4 and the sign property for $\partial_{d}$ discussed above.

Define $f_{i}=\left|\Delta_{i}\right|$ and $f_{i}^{\circ}=\left|\Delta_{i}^{\circ}\right|, i=0, \ldots, d$. Using Proposition 2.6, we can compute the Euler characteristic $\chi$ of the complex $\mathscr{C}_{m}^{r}$.

THEOREM 3.3. For $m \geq r \geq 0$,

$$
(-1)^{d} \chi\left(\mathscr{C}_{m}^{r}\right)=\left(\begin{array}{c}
m+d \\
d
\end{array}\right) f_{d}+\sum_{i=0}^{d-1}(-1)^{d-i}\left[\sum_{j=0}^{r}\left(\begin{array}{c}
m+i-j \\
i
\end{array}\right)\left(\begin{array}{c}
j+d-i-1 \\
j
\end{array}\right)\right] f_{i}^{\circ} \text {. }
$$

Let $\chi(\Delta, \partial \Delta)$ denote the Euler characteristic of the pair $(\Delta, \partial \Delta)$. From the note following the proof of Proposition 2.6, we have

PROPOSITION 3.4. If $r \geq m$,

$$
\chi\left(\mathscr{C}_{m}^{r}\right)=\left(\begin{array}{c}
m+d \\
d
\end{array}\right) \chi(\Delta, \partial \Delta)
$$

We consider now the short exact sequences of complexes induced by two special maps. These, in turn, induce in the usual way long exact sequences in homology (see, e.g. [21, p. 182]) that, under certain conditions, allow us to read off useful information. The first of these is straightforward. 
Proposition 3.5. For $r \geq 0$, the canonical projection $P_{m} \rightarrow P_{m} / P_{m} \cap I_{\sigma}^{r+1}$, for $\sigma \in \Delta$, induces an exact sequence of complexes of the form

$$
0 \rightarrow \mathscr{K}_{m}^{r} \rightarrow C\left(\Delta, \partial \Delta ; P_{m}\right) \rightarrow \mathscr{C}_{m}^{r} \rightarrow 0
$$

where $C\left(\Delta, \partial \Delta ; P_{m}\right)$ is the usual complex to compute $H_{*}\left(\Delta, \partial \Delta ; P_{m}\right)$ and $\mathscr{K}_{m}^{r}$ is the complex

$$
0 \rightarrow 0 \rightarrow \bigoplus_{\tau \in \Delta_{d-1}^{\circ}}\left(P_{m} \cap I_{\tau}^{r+1}\right) \rightarrow \cdots \rightarrow \bigoplus_{v \in \Delta_{0}^{\circ}}\left(P_{m} \cap I_{\nu}^{r+1}\right) \rightarrow 0 .
$$

COROLLARY 3.6. If $\Delta$ is a connected complex, then $H_{0}\left(\mathscr{C}_{m}^{r}\right)=0$ for all $m \geq 0$ and $r \geq 0$.

PROOF. For a connected complex we have $H_{0}(\Delta, \partial \Delta)=0$, and so, by the universal coefficient theorem $[\mathbf{2 1}], H_{0}\left(\Delta, \partial \Delta ; P_{m}\right)=0$. Now the short exact sequence of complexes in Proposition 3.5 induces a long exact sequence in homology, the tail of which is

$$
\cdots \rightarrow H_{0}\left(\mathscr{K}_{m}^{r}\right) \rightarrow H_{0}\left(\Delta, \partial \Delta ; P_{m}\right) \rightarrow H_{0}\left(\mathscr{C}_{m}^{r}\right) \rightarrow 0 .
$$

We see directly that $H_{0}\left(\Delta, \partial \Delta ; P_{m}\right)=0$ implies $H_{0}\left(\mathscr{C}_{m}^{r}\right)=0$.

Now consider the inclusion $P_{m} \hookrightarrow P_{m+1}$ of polynomials of degree at most $m$ into those of degree at most $m+1$. If $I$ is any ideal in $A=\mathbf{R}\left[x_{1}, \ldots, x_{d}\right]$, then $P_{m} \hookrightarrow P_{m+1}$ induces an exact sequence of vector spaces

$$
0 \rightarrow P_{m} /\left(P_{m} \cap I\right) \rightarrow P_{m+1} /\left(P_{m+1} \cap I\right) \rightarrow P_{m+1} /\left(P_{m}+\left(P_{m+1} \cap I\right)\right) \rightarrow 0,
$$

where the first map takes the equivalence class $\bmod \left(P_{m} \cap I\right)$ of $p \in P_{m}$ to the class of $p \bmod \left(P_{m+1} \cap I\right)$, and the second map is similarly defined. That the sequence is exact follows from the isomorphisms of abelian groups $B \subset A \supset C$,

$$
A /(B+C) \cong(A / C) /(B+C / C) \text { and } B+C / C \cong B / B \cap C,
$$

applied to $A=P_{m+1}, B=P_{m}$ and $C=P_{m+1} \cap I$. Applying this when $I=I_{\sigma}^{r+1}$, $\sigma \in \Delta$, we get the following.

PROPOSITION 3.7. The inclusion $P_{m} \hookrightarrow P_{m+1}$ induces an exact sequence of complexes

where $\mathscr{C} \mathscr{K}_{m+1}^{r}$ is the complex

$$
0 \rightarrow \mathscr{C}_{m}^{r} \rightarrow \mathscr{C}_{m+1}^{r} \rightarrow \mathscr{C} \mathscr{K}_{m+1}^{r} \rightarrow 0
$$

$$
\begin{aligned}
0 \rightarrow \bigoplus_{\sigma \in \Delta_{d}} P_{m+1} / P_{m} & \rightarrow \bigoplus_{\tau \in \Delta_{d-1}^{\circ}} P_{m+1} /\left(P_{m}+\left(P_{m+1} \cap I_{\tau}^{r+1}\right)\right) \rightarrow \cdots \\
& \rightarrow \bigoplus_{\nu \in \Delta_{0}^{\circ}} P_{m+1} /\left(P_{m}+\left(P_{m+1} \cap I_{v}^{r+1}\right)\right) \rightarrow 0
\end{aligned}
$$

with boundary maps given as in (3.1).

Finally, we note that for $m \geq r$, the last term in the complex $\mathscr{C} \mathscr{K}_{m+1}^{r}$ will always be 0 . This follows from

PROPOSITION 3.8. For $m \geq r$

$$
P_{m+1} /\left(P_{m}+\left(P_{m+1} \cap I_{v}^{r+1}\right)\right)=0 .
$$

PROOF. As before, we can assume without loss of generality that $v=0$ and so $I_{v}=\left\langle x_{1}, \ldots, x_{d}\right\rangle$. In this case $P_{m}+\left(P_{m+1} \cap I_{v}^{r+1}\right)=P_{m+1}$. 
4. The case $d=2$. We now specialize the results of the previous section to the case of a 2-dimensional complex $\Delta$ embedded in the plane. The complex $\mathscr{C}_{m}^{r}$ in (3.1) becomes

$$
\mathscr{C}_{m}^{r}: 0 \rightarrow \bigoplus_{\sigma \in \Delta_{2}} P_{m} \stackrel{\partial_{2}}{\longrightarrow} \bigoplus_{e \in \Delta_{1}^{\circ}} P_{m} /\left(P_{m} \cap I_{e}^{r+1}\right) \stackrel{\partial_{1}}{\longrightarrow} \bigoplus_{v \in \Delta_{0}^{\circ}} P_{m} /\left(P_{m} \cap I_{v}^{r+1}\right) \rightarrow 0 .
$$

By Lemma 2.1, we have that $I_{e}=\left\langle l_{e}\right\rangle$, when $l_{e}$ is a nontrivial affine function which vanishes on the edge $e$. If $v=\left(v_{1}, v_{2}\right)$ then by Proposition 2.5, $I_{v}=\left\langle x-v_{1}, y-v_{2}\right\rangle$.

Specializing Theorem 3.3 to $d=2$ gives

$$
\begin{aligned}
\chi\left(\mathscr{C}_{m}^{r}\right) & =\left(\begin{array}{c}
m+2 \\
2
\end{array}\right) f_{2}-\left[\sum_{j=0}^{r}\left(\begin{array}{c}
m+1-j \\
1
\end{array}\right)\left(\begin{array}{l}
j \\
j
\end{array}\right)\right] f_{1}^{\circ}+\left[\sum_{j=0}^{r}\left(\begin{array}{c}
m-j \\
0
\end{array}\right)\left(\begin{array}{c}
j+1 \\
j
\end{array}\right)\right] f_{0}^{\circ} \\
& =\left(\begin{array}{c}
m+2 \\
2
\end{array}\right) f_{2}-\left[\sum_{j=0}^{r}(m+1-j)\right] f_{1}^{\circ}+\left[\sum_{j=0}^{r}(j+1)\right] f_{0}^{\circ} \\
& =\left(\begin{array}{c}
m+2 \\
2
\end{array}\right) f_{2}-\left[\left(\begin{array}{c}
m+2 \\
2
\end{array}\right)-\left(\begin{array}{c}
m-r+1 \\
2
\end{array}\right)\right] f_{1}^{\circ}+\left(\begin{array}{c}
r+2 \\
2
\end{array}\right) f_{0}^{\circ},
\end{aligned}
$$

for $m \geq r$. From this we can obtain a lower bound on the dimension of $S_{m}^{r}(\Delta)$ for a large class of complexes $\Delta$.

THEOREM 4.3. If $\Delta \subset \mathbf{R}^{2}$ is a connected 2-manifold, with $f_{2}$ triangles, $f_{1}^{\circ}$ interior edges and $f_{0}^{\circ}$ interior vertices, then for $m \geq r \geq 0$

$$
\operatorname{dim} S_{m}^{r}(\Delta) \geq\left(\begin{array}{c}
m+2 \\
2
\end{array}\right) f_{2}-\left[\left(\begin{array}{c}
m+2 \\
2
\end{array}\right)-\left(\begin{array}{c}
m-r+1 \\
2
\end{array}\right)\right] f_{1}^{\circ}+\left(\begin{array}{c}
r+2 \\
2
\end{array}\right) f_{0}^{\circ} .
$$

Proof. We have

$$
\operatorname{dim} H_{2}\left(\mathscr{C}_{m}^{r}\right)-\operatorname{dim} H_{1}\left(\mathscr{C}_{m}^{r}\right)+\operatorname{dim} H_{0}\left(\mathscr{C}_{m}^{r}\right)=\chi\left(\mathscr{C}_{m}^{r}\right) .
$$

By Corollary 3.6, $H_{0}\left(\mathscr{C}_{m}^{r}\right)=0$ and so by Theorem 3.2

$$
\operatorname{dim} S_{m}^{r}(\Delta)=\chi\left(\mathscr{C}_{m}^{r}\right)+\operatorname{dim} H_{1}\left(\mathscr{C}_{m}^{r}\right) .
$$

Using (4.2) and the fact that $\operatorname{dim} H_{1}\left(\mathscr{C}_{m}^{r}\right) \geq 0$, we get the desired inequality.

We note here that by (4.4), the error in the inequality of Theorem 4.3 is measured by the space $H_{1}\left(\mathscr{C}_{m}^{r}\right)$.

Using the relation $\chi(\Delta, \partial \Delta)=f_{2}-f_{1}^{\circ}+f_{0}^{\circ}$, the inequality in Theorem 4.3 can be rewritten as

$$
\operatorname{dim} S_{m}^{r}(\Delta) \geq\left(\begin{array}{c}
m+2 \\
2
\end{array}\right) \chi(\Delta, \partial \Delta)+\left(\begin{array}{c}
m-r+1 \\
2
\end{array}\right) f_{1}^{\circ}-\left[\left(\begin{array}{c}
m+2 \\
2
\end{array}\right)-\left(\begin{array}{c}
r+2 \\
2
\end{array}\right)\right] f_{0}^{\circ} .
$$

In the case that $\Delta$ is a 2-disk (and so $\chi(\Delta, \partial \Delta)=1$ ), (4.5) is a weaker form of the inequality of Schumaker [19, Theorem 3.1]. (The proof in [19] involves a construction that seems to assume implicitly that $\Delta$ triangulates a disk anyway.)

Specializing Theorem 4.3 to the case $r=1$, we have for $m \geq 1$,

$$
\operatorname{dim} S_{m}^{1}(\Delta) \geq\left(\begin{array}{c}
m+2 \\
2
\end{array}\right) f_{2}-(2 m+1) f_{1}^{\circ}+3 f_{0}^{\circ} .
$$


It is the conjecture of Strang that this inequality holds as an equation for $m \geq 2$ for generic triangulations $\Delta$. This was proved for $m \geq 5$ by Morgan and Scott [17] and for $m=4$ by Alfeld, Piper and Schumaker [3] for any 2-manifold $\Delta$.

In fact, $[\mathbf{1 7}]$ and [3] prove an equality in which the right-hand side of (4.6) is augmented by a correction term that counts the number of degree 4 vertices whose edges lie on only two straight lines. A similar term, involving a measure of the local deficiency in slope over all vertices, appears in the inequality of Schumaker for each $r$. By (4.4), these correction terms are captured somehow by the spaces $H_{1}\left(\mathscr{C}_{m}^{r}\right)$. It is important to note that for generic embeddings, the $r=1$ correction term vanishes; this is not so for $r>1$. It is our aim to show, in fact, that $H_{1}\left(\mathscr{C}_{m}^{1}\right)=0$, for generic embeddings of a 2-manifold $\Delta^{*}$, for all $m \geq 2$, thereby proving the conjecture of Strang.

Note that by the remark immediately preceding Theorem $2.4, \operatorname{dim} S_{r}^{r}(\Delta)=\left(\begin{array}{c}r+2 \\ 2\end{array}\right)$ for a connected 2-manifold $\Delta \subset \mathbf{R}^{2}$, so by (4.4) we have

$$
\begin{aligned}
\operatorname{dim} H_{1}\left(\mathscr{C}_{r}^{r}\right) & =\left(\begin{array}{c}
r+2 \\
2
\end{array}\right)-\chi\left(\mathscr{C}_{r}^{r}\right)=\left(\begin{array}{c}
r+2 \\
2
\end{array}\right)(1-\chi(\Delta, \partial \Delta)) \\
& =\left(\begin{array}{c}
r+2 \\
2
\end{array}\right)(1-\chi(\Delta))=\left(\begin{array}{c}
r+2 \\
2
\end{array}\right) \beta_{1},
\end{aligned}
$$

where $\beta_{1}$ is the number of "holes" in $\Delta$. Thus when $\Delta$ is a disk, $\chi(\Delta)=\chi(\Delta, \partial \Delta)=$ 1 , and we have the following.

PROPOSITION 4.8. If $\Delta$ is a 2-disk, then for all $r, H_{1}\left(\mathscr{C}_{r}^{r}\right)=0$.

In particular, $H_{1}\left(\mathscr{C}_{1}^{1}\right)=0$ for any embedding of a 2-disk $\Delta$.

Recall the short exact sequence of complexes induced by the inclusion $P_{m} \hookrightarrow$ $P_{m+1}$ in Proposition 3.7,

$$
0 \rightarrow \mathscr{C}_{m}^{r} \rightarrow \mathscr{C}_{m+1}^{r} \rightarrow \mathscr{C} \mathscr{K}_{m+1}^{r} \rightarrow 0
$$

where for $m \geq r \mathscr{C} \mathscr{K}_{m+1}^{r}$ is the complex

$$
\mathscr{C} \mathscr{K}_{m+1}^{r}: 0 \rightarrow \bigoplus_{\sigma \in \Delta_{2}} P_{m+1} / P_{m} \stackrel{\delta_{m+1}^{r}}{\longrightarrow} \bigoplus_{e \in \Delta_{1}^{\circ}} P_{m+1} /\left(P_{m}+\left(P_{m+1} \cap I_{e}^{r+1}\right)\right) \rightarrow 0 \rightarrow 0
$$

by Proposition 3.8. The sequence (4.9) induces a long exact sequence of homology, part of which is

$$
\cdots \rightarrow H_{1}\left(\mathscr{C}_{m}^{r}\right) \rightarrow H_{1}\left(\mathscr{C}_{m+1}^{r}\right) \rightarrow H_{1}\left(\mathscr{C} \mathscr{K}_{m+1}^{r}\right) \rightarrow H_{0}\left(\mathscr{C}_{m}^{r}\right) \rightarrow \cdots
$$

By Corollary 3.6, $H_{0}\left(\mathscr{C}_{m}^{r}\right)=0$ for a connected 2-complex $\Delta$, so this gives an exact sequence of vector spaces

$$
H_{1}\left(\mathscr{C}_{m}^{r}\right) \rightarrow H_{1}\left(\mathscr{C}_{m+1}^{r}\right) \rightarrow H_{1}\left(\mathscr{C} \mathscr{K}_{m+1}^{r}\right) \rightarrow 0
$$

PROPOSITION 4.11. For a connected complex $\Delta \subset \mathbf{R}^{2}$, we have for any $m \geq r$,

$$
H_{1}\left(\mathscr{C}_{m}^{r}\right)=0 \text { implies } H_{1}\left(\mathscr{C}_{m+1}^{r}\right) \cong H_{1}\left(\mathscr{C} \mathscr{K}_{m+1}^{r}\right) \text {, }
$$

and

$$
H_{1}\left(\mathscr{C} \mathscr{K}_{m+1}^{r}\right)=0 \text { implies } \operatorname{dim} H_{1}\left(\mathscr{C}_{m+1}^{r}\right) \leq \operatorname{dim} H_{1}\left(\mathscr{C}_{m}^{r}\right) .
$$

ProOF. This follows directly from the sequence (4.10).

An immediate corollary of Propositions 4.8 and 4.11 is the following. 
COROLlaRY 4.12. For a 2-disk $\Delta \subset \mathbf{R}^{2}$,

$$
H_{1}\left(\mathscr{C}_{r+1}^{r}\right) \cong H_{1}\left(\mathscr{C} \mathscr{K}_{r+1}^{r}\right)
$$

In order to achieve our aim of showing that $H_{1}\left(\mathscr{C}_{m}^{1}\right)=0$, for $m \geq 2$, for generic embeddings of a 2-manifold $\Delta$, we must show that, generically, $H_{1}\left(\mathscr{C} \mathscr{K}_{m}^{1}\right)=0$ for $m \geq 2$. This will enable us, in the case of $\Delta$ a 2-disk, to bootstrap the vanishing of $H_{1}\left(\mathscr{C}_{1}^{1}\right)$ to give the vanishing of $H_{1}\left(\mathscr{C}_{m}^{1}\right)$ for all $m \geq 1$. When $\Delta$ is a general 2-manifold, $H_{1}\left(\mathscr{C}_{1}^{1}\right)$ is not necessarily zero by (4.7). In this case the vanishing of $H_{1}\left(\mathscr{C}_{2}^{1}\right)$ (when it occurs) will then imply, by $(4.11 .1)$, the vanishing of $H_{1}\left(\mathscr{C}_{m}^{1}\right)$ for $m \geq 3$.

In the next section we show that the spaces $H_{1}\left(\mathscr{C} \mathscr{K}_{m}^{1}\right)$ vanish generically for $m \geq 2$, for all 2-manifolds in the plane. We devote the remainder of this section to proving the following result.

PROPOSITION 4.13. If $H_{1}\left(\mathscr{C}_{2}^{1}\right)=0$ for generic embeddings of 2 -disks in the plane, then it holds also for generic embeddings of any 2-manifold.

ProOF. By (4.4) we have

$$
\operatorname{dim} S_{2}^{1}(\Delta)-\operatorname{dim} H_{1}\left(\mathscr{C}_{2}^{1}\right)=6 f_{2}-5 f_{1}^{\circ}+3 f_{0}^{\circ} .
$$

By hypothesis, then, if $\Delta$ is a generically embedded 2-disk, we have

$$
\operatorname{dim} S_{2}^{1}(\Delta)=6 f_{2}-5 f_{1}^{\circ}+3 f_{0}^{\circ} .
$$

To prove the result, we must show that (4.14) continues to hold for any generically embedded 2-manifold $\Delta$. The proof is by induction on $\beta_{1}(\Delta)$, the number of holes in $\Delta$; the case $\beta_{1}(\Delta)=0$ is the hypothesis. If $\beta_{1}(\Delta)>0$, we have a further induction on the number of edges bounding a given hole. If the hole is triangular, then filling it in with a single triangle results in a complex $\Delta^{\prime}$ with one fewer hole. Since $\Delta^{\prime}$ has one more triangle, 3 more interior edges and 3 more interior vertices, to prove (4.14) for $\Delta^{\prime}$, we must show $\operatorname{dim} S_{2}^{1}(\Delta)=\operatorname{dim} S_{2}^{1}\left(\Delta^{\prime}\right)$. If the hole has more than three edges, then adding a triangle joining any three successive vertices gives a complex $\Delta^{\prime}$ with a hole having one fewer edge and with two additional interior edges and one additional interior vertex. In this case, proving (4.14) for $\Delta^{\prime}$ requires showing

$$
\operatorname{dim} S_{2}^{1}(\Delta)-\operatorname{dim} S_{2}^{1}\left(\Delta^{\prime}\right)=1
$$

In either case, consider the short exact sequence of complexes

$$
0 \rightarrow \mathscr{K} \rightarrow \mathscr{C}_{2}^{1}\left(\Delta^{\prime}\right) \rightarrow \mathscr{C}_{2}^{1}(\Delta) \rightarrow 0
$$

induced by the canonical projection

$$
\bigoplus_{\sigma \in \Delta_{2}^{\prime}} P_{2} \rightarrow \bigoplus_{\sigma \in \Delta_{2}} P_{2} \rightarrow 0
$$

If the new triangle in $\Delta^{\prime}$ is denoted by $\tau$, then the kernel complex $\mathscr{K}$ is given by

$$
\mathscr{K}_{1}: 0 \rightarrow P \stackrel{\partial_{2}}{\longrightarrow} \bigoplus_{i=1}^{3} P_{2} /\left(P_{2} \cap i_{e_{i}}^{2}\right) \stackrel{\partial_{1}}{\longrightarrow} \bigoplus_{i=1}^{3} P_{2} /\left(P_{2} \cap I_{v_{i}}^{2}\right) \rightarrow 0
$$

in the case where $\tau$ has 3 edges in common with $\Delta$, and by

$$
\mathscr{K}_{2}: 0 \rightarrow P_{2} \stackrel{\partial_{2}}{\longrightarrow} P_{2} /\left(P_{2} \cap I_{e_{1}}^{2}\right) \oplus P_{2} /\left(P_{2} \cap I_{e_{2}}^{2}\right) \stackrel{\partial_{1}}{\longrightarrow} P_{2} /\left(P_{2} \cap I_{v}^{2}\right) \rightarrow 0
$$


in the other case. It is a straightforward calculation to verify that $H_{i}\left(\mathscr{K}_{1}\right)=0$ for $i=0,1,2$ and that $H_{i}\left(\mathscr{K}_{2}\right)=0$ for $i=0,2$ while $\operatorname{dim} H_{1}\left(\mathscr{K}_{2}\right)=1$ for generic embeddings of $\tau$ (not all vertices on a line). For example, to check the assertions about $H_{2}\left(\mathscr{K}_{j}\right)$, observe that it is the space spanned by quadratic polynomials divisible by the squares of linear forms defining the three edges of $\tau$ in the case $j=1$ and by those defining two edges in the case $j=2$. In either case, there are none.

Now the short exact sequence (4.15) induces a long exact sequence in homology, the beginning of which is

$$
0 \rightarrow H_{2}(\mathscr{K}) \rightarrow S_{2}^{1}\left(\Delta^{\prime}\right) \rightarrow S_{2}^{1}(\Delta) \rightarrow H_{1}(\mathscr{K}) \rightarrow H_{1}\left(\mathscr{C}_{2}^{1}\left(\Delta^{\prime}\right)\right) .
$$

To complete the proof, note that in the case of a triangular hole, this sequence together with $H_{i}\left(\mathscr{K}_{1}\right)=0$ for $i=1,2$ shows $S_{2}^{1}\left(\Delta^{\prime}\right) \cong S_{2}^{1}(\Delta)$ (giving a generalization of a result of Whiteley [29, Theorem 2.4]). In the other case, the induction hypothesis on $\Delta^{\prime}$ gives $H_{1}\left(\mathscr{C}_{2}^{1}\left(\Delta^{\prime}\right)\right)=0$ and so the sequence together with $H_{2}\left(\mathscr{K}_{2}\right)=0$ gives an exact sequence

$$
0 \rightarrow S_{2}^{1}\left(\Delta^{\prime}\right) \rightarrow S_{2}^{1}(\Delta) \rightarrow H_{1}\left(\mathscr{K}_{2}\right) \rightarrow 0 .
$$

Together with $\operatorname{dim} H_{1}\left(\mathscr{K}_{2}\right)=1$, this completes the proof.

5. Generic triangulations. In this section, we complete the development necessary to prove Strang's conjecture by showing that $H_{1}\left(\mathscr{C}_{m}^{1}\right)=0, m \geq 2$, for generic embeddings of 2-disks in the plane. As discussed in the previous section, this will follow if we can show $H_{1}\left(\mathscr{C} \mathscr{K}_{m}^{1}\right)=0$ generically for $m \geq 2$. We accomplish this by reducing this to a question of showing that a certain matrix has generically independent columns. This last question was recently settled by Whiteley [28].

For now, we let $\Delta$ be any 2-manifold (with boundary) embedded in $\mathbf{R}^{2}$. Recall for general $r \geq 0$ and $m \geq r+1$, the complex $\mathscr{C} \mathscr{K}_{m}^{r}$ is given by

$$
\mathscr{C} \mathscr{K}_{m}^{r}: 0 \rightarrow \bigoplus_{\sigma \in \Delta_{2}} P_{m} / P_{m-1} \stackrel{\delta_{m}^{r}}{\longrightarrow} \bigoplus_{e \in \Delta_{1}^{\circ}} P_{m} /\left(P_{m-1}+\left(P_{m} \cap I_{e}^{r+1}\right)\right) \rightarrow 0 \rightarrow 0
$$

so the statement $H_{1}\left(\mathscr{C} \mathscr{K}_{m}^{r}\right)=0$ is equivalent to the surjectivity of the map $\delta_{m}^{r}$. We show first that it is enough to show that $\delta_{r+1}^{r}$ is surjective.

LEMMA 5.1. For $m \geq r+1, \delta_{m}^{r}$ surjective implies $\delta_{m+1}^{r}$ is also surjective.

PROOF. To show $\delta_{m+1}^{r}$ is surjective, we must show that for each $e \in \Delta_{1}^{\circ}$ and each monomial $p$ in $P_{m+1}$ of degree $m+1$, we have a $q=\left(\bar{q}_{\sigma}\right)_{\sigma \in \Delta_{2}}$ in $\bigoplus_{\sigma \in \Delta_{2}} P_{m+1} / P_{m}$ so that

$$
\delta_{m+1}^{r}(q)_{e}-p \in P_{m}+\left(P_{m+1} \cap I_{e}^{r+1}\right)
$$

and for $e \neq e^{\prime}$,

$$
\delta_{m+1}^{r}(q)_{e^{\prime}} \in P_{m}+\left(P_{m+1} \cap I_{e^{\prime}}^{r+1}\right) .
$$

Suppose $p=x_{1} p^{\prime}$, where $p^{\prime} \in P_{m}$. By the surjectivity of $\delta_{m}^{r}$ there is a $q^{\prime}=$ $\left(\bar{q}_{\sigma}^{\prime}\right)_{\sigma \in \Delta_{2}}, \bar{q}_{\sigma}^{\prime} \in P_{m} / P_{m-1}$, so that

$$
\delta_{m}^{r}\left(q^{\prime}\right)_{e}-p^{\prime} \in P_{m-1}+\left(P_{m} \cap I_{e}^{r+1}\right)
$$

and for $e \neq e^{\prime}$

$$
\delta_{m}^{r}\left(q^{\prime}\right)_{e^{\prime}} \in P_{m-1}+\left(P_{m} \cap I_{e}^{r+1}\right)
$$


Let $q_{\sigma}^{\prime}$ represent the class $\bar{q}_{\sigma}^{\prime}$ for each $\sigma \in \Delta_{2}$. Define $q=\left(\overline{x_{1} q_{\sigma}^{\prime}}\right)_{\sigma \in \Delta_{2}}$. By the definition of the boundary map, for each $e^{\prime} \in \Delta_{1}^{0}$

$$
\delta_{m+1}^{r}(q)_{e^{\prime}}=x_{1} \delta_{m}^{r}\left(q^{\prime}\right)_{e^{\prime}}
$$

and so (5.2) and (5.3) hold for this $q$.

Consider now the case $r=1$. By the previous result, we need only consider the map

$$
\bigoplus_{\sigma \in \Delta_{2}} P_{2} / P_{1} \stackrel{\delta_{2}^{1}}{\longrightarrow} \bigoplus_{e \in \Delta_{1}^{\circ}} P_{2} /\left(P_{1}+\left(P_{2} \cap I_{e}^{2}\right)\right)
$$

Here $\delta_{2}^{1}$ is induced by the usual boundary map $\partial_{2}$ obtained by orienting each 2simplex $\sigma$ counterclockwise, and directing the edges $e \in \Delta_{1}^{0}$ arbitrarily. The sign of $e$ in $\partial_{2}(\sigma)$ is \pm 1 if $e \subset \sigma$, depending upon whether or not the direction of $e$ agrees with the orientation of $\sigma$, and is 0 if $e \not \subset \sigma$.

Each component of $\delta_{2}^{1}$ involves the projections

$$
P_{2} / P_{1} \rightarrow P_{2} /\left(P_{1}+\left(P_{2} \cap I_{e}^{2}\right)\right)
$$

which we consider first. The vector space $P_{2} / P_{1}$ has as basis the equivalence classes of the monomials $x^{2}, y^{2}$ and $2 x y$ (the factor 2 being included for convenience). Suppose $I_{e}=\left\langle l_{e}\right\rangle$, where $l_{e}$ is an affine function vanishing on the edge $e$. If $e$ has endpoints with coordinates $\left(x_{1}, y_{1}\right)$ and $\left(x_{2}, y_{2}\right)$, then $l_{e}$ can be taken to be the affine function given by the determinant

$$
l_{e}=\left|\begin{array}{ccc}
x & y & 1 \\
x_{1} & y_{1} & 1 \\
x_{2} & y_{2} & 1
\end{array}\right|=\left(y_{1}-y_{2}\right) x-\left(x_{1}-x_{2}\right) y+\left(x_{1} y_{2}-y_{1} x_{2}\right)
$$

Thus $I_{e}^{2}=\left\langle l_{e}^{2}\right\rangle$, where

$$
l_{e}^{2}=\left(y_{1}-y_{2}\right)^{2} x^{2}-\left(y_{1}-y_{2}\right)\left(x_{1}-x_{2}\right)(2 x y)+\left(x_{1}-x_{2}\right)^{2} y^{2}+\text { terms in } P_{1},
$$

and so in $P_{2} /\left(P_{1}+\left(P_{2} \cap I_{e}^{2}\right)\right)$ we have that (for equivalence classes)

$$
2 x y=\frac{y_{1}-y_{2}}{x_{1}-x_{2}} x^{2}+\frac{x_{1}-x_{2}}{y_{1}-y_{2}} y^{2} .
$$

If we take as basis for this space the classes of $x^{2}$ and $y^{2}$, we get that the map (5.5) has the matrix

$$
B_{3}=\left(\begin{array}{cc}
1 & 0 \\
0 & 1 \\
m_{e} & 1 / m_{e}
\end{array}\right)
$$

where $m_{e}=\left(y_{1}-y_{2}\right) /\left(x_{1}-x_{2}\right)$ is the slope of the edge $e$.

We assume from now on that if the vertices of $\Delta$ have coordinates $\left(x_{1}, y_{1}\right),\left(x_{2}, y_{2}\right)$, $\ldots,\left(x_{n}, y_{n}\right)$ then the polynomial $V(\mathbf{x}, \mathbf{y}) \neq 0$, where

$$
V(\mathbf{x}, \mathbf{y})=\prod_{i<j}\left(x_{i}-x_{j}\right)\left(y_{i}-y_{j}\right)
$$

Now the matrix $M$ of the usual boundary map $\partial_{2}$ on $\Delta$ has rows indexed by $\sigma \in \Delta_{2}$ and columns indexed by $e \in \Delta_{1}^{\circ}$, and the $(\sigma, e)$ entry is the sign of $e$ in $\partial_{2}(\sigma)$ as discussed above. Equivalently, $M$ is the vertex-edge incidence matrix of 
the directed graph $G(\Delta)$ whose vertices are the triangles in $\Delta$, where an edge exists between triangle $\sigma$ and $\sigma^{\prime}$ precisely when $\sigma \cap \sigma^{\prime}$ is an (interior) edge of $\Delta$. (That is, $G(\Delta)$ is the planar dual of the 1-skeleton of $\Delta$, restricted to duals of edges in $\Delta_{1}^{\circ}$.) The orientation of the edges in $G(\Delta)$ is such that they can be viewed as 90 degree counterclockwise rotations of their dual edges in $\Delta_{1}^{\circ}$. For $e \in \Delta_{1}^{\circ}$, we will often refer to the corresponding dual edge in $G(\Delta)$ also as $e$.

The matrix $D$ of the map $\delta_{2}^{1}$ in (5.4) is obtained from $M$ by substitution for each entry in column $e$ by $\pm B_{e}$ or a $3 \times 2$ block of zeros, depending on whether the entry in question is \pm 1 or 0 . We will view $D=D(\mathbf{x}, \mathbf{y})$ as a matrix whose entries are rational functions of the $x_{i}$ and $y_{i}$. To show that $\delta_{2}^{1}$ is generically surjective is to show that for generic embeddings of $\Delta, D$ has linearly independent columns; that is, considered as a matrix over the field of rational functions on $2 n$ indeterminates, $F=\mathbf{R}\left(x_{1}, y_{1}, \ldots, x_{n}, y_{n}\right), D$ has linearly independent columns.

Multiplying columns of $D$ by the appropriate $x_{i}-x_{j}$ or $y_{i}-y_{j}$, we get an equivalent matrix $D^{\prime}$ over $F$, which is obtained from $M$ by the substitution of \pm 1 or 0 times the block

$$
\left(\begin{array}{cc}
x_{i}-x_{j} & 0 \\
0 & y_{i}-y_{j} \\
y_{i}-y_{j} & x_{i}-x_{j}
\end{array}\right)
$$

III coiumn $e$, where $e$ is dual to the edge between $\left(x_{i}, y_{i}\right)$ and $\left(x_{j}, y_{j}\right)$. It is this so-called "spline matrix" that is shown by Whiteley [28] to have generically independent columns. His proof is an induction on the number of interior vertices; the inductive step consists of shrinking an interior edge and dealing with the resulting degenerated triangles. This matrix is related to matrices arising in structural rigidity theory [26].

Thus, combining the above discussion with Lemma 5.1, we have the following result.

LEMMA 5.7. For generic embeddings of any triangulated 2-manifold $\Delta$ in $\mathbf{R}^{2}$, $H_{1}\left(\mathscr{C}, \mathscr{K}_{m}^{1}\right)=0$ for $m \geq 2$.

We can now formally assemble the proof of Strang's conjecture. $\mathbf{R}^{2}$

THEOREM 5.8. For generic embeddings of any triangulated 2-manifold $\Delta$ in

$$
\operatorname{dim} S_{m}^{1}(\Delta)=\left(\begin{array}{c}
m+2 \\
2
\end{array}\right) f_{2}-(2 m+1) f_{1}^{\circ}+3 f_{0}^{\circ} .
$$

ProOF. By (4.4), we must show $H_{1}\left(\mathscr{C}_{m}^{r}\right)=0$ for all $m \geq 2$. We assume first that $\Delta$ is a 2 -disk. Then by Proposition $4.8, H_{1}\left(\mathscr{C}_{1}^{1}\right)=0$, so by repeated uses of (4.11.1) and Lemma 5.7, we conclude $H_{1}\left(\mathscr{C}_{m}^{1}\right)=0$ for all $m \geq 2$ as well. To complete the proof for a general 2-manifold $\Delta$, by the first part of the proof and Proposition $4.13, H_{1}\left(\mathscr{C}_{2}^{1}\right)=0$ for generic embeddings of $\Delta$. Thus again by repeated uses of (4.11.1) and Lemma 5.7, we get $H_{1}\left(\mathscr{C}_{m}^{1}\right)=0$ for all $m \geq 2$.

Note that for a 2-disk $\Delta$, an embedding is bad for $S_{m}^{1}(\Delta)$ for some $m \geq 2$ (i.e., gives a dimension strictly larger than that in the theorem) precisely when it is bad for $m=2$. That is, if a triangulation admits the right number of $C^{1}$ quadratics, then it has the right number for all other degrees. To see this, recall that by Corollary $4.12, H_{1}\left(\mathscr{C}_{2}^{1}\right) \cong H_{1}\left(\mathscr{C} \mathscr{K}_{2}^{1}\right)$, so if $H_{1}\left(\mathscr{C}_{2}^{1}\right)=0$ we have that the map $\delta_{2}^{1}$ 
in (5.4) is surjective and so, by Lemma $5.1, \delta_{m}^{1}$ is surjective for all $m \geq 2$. This gives the conclusion of Lemma 5.7 for $\Delta$.

Morgan and Scott [17] have shown that for any planar embedding of a 2-manifold $\Delta$, and for $m \geq 5$, the dimension of $S_{m}^{1}(\Delta)$ is equal to the value given in Theorem 5 plus the number of quadrilaterals in the complex which are triangulated by crossing straight lines. Alfeld, Piper and Schumaker [3] have shown this to hold for $m=4$ as well, while Schumaker [19] has shown this to be a lower bound for $m \geq 2$. It is easy to see that for a single quadrilateral triangulated with a single interior vertex, the nongeneric positions are exactly those in which the four interior edges lie on two straight lines (aside from trivial degeneracies in which all the vertices of some triangle lie on some line). Thus, for such embeddings, we get one extra quadratic which causes the count to be one higher for all $m$.

On the other hand, the triangulation of a triangle with three interior vertices given by the 1-skeleton of the 3-dimensional octahedron has been shown by Morgan and Scott (unpublished [18], see also [13], [5] or [2]) to have a nongeneric embedding resulting in one more quadratic than predicted by Strang's formula. However, there are no crossing diagonals in this embedding, and so by the above results, the formula is correct for $m \geq 4$. Direct calculation shows it to be correct for $m=3$ as well [2].

From all this, we see that the behavior of the $S_{m}^{1}(\Delta)$ in nongeneric cases can be fairly subtle. The deviations of the dimensions of these spaces from Strang's formula is completely described by the spaces $H_{1}\left(\mathscr{C}_{m}^{1}\right)$, and perhaps further study of these will shed more light on these issues.

6. Variations and extensions. We discuss here a few related problems which can be approached by variations in the techniques in this paper and some of the new problems that arise. Most of these variations have not been investigated very deeply.

A problem which has considerable geometric interest (e.g., see $[\mathbf{1 3}, \mathbf{2 7}]$ ) is the study of continuous piecewise linear functions over not-necessarily-simplicial decompositions of a $d$-dimensional region in $\mathbf{R}^{d}$. For $d=2$, the techniques of $\S \S 3$ and 4 apply directly and we get the relation

$$
\operatorname{dim} S_{1}^{\circ}(\Delta)=3 f_{2}-2 f_{1}^{\circ}+f_{0}^{\circ}+\operatorname{dim} H_{1}\left(\mathscr{C}_{1}^{0}\right)
$$

from (4.2) and (4.4). In particular, when $\Delta$ is a 2-disk, $H_{1}\left(\mathscr{C}_{0}^{0}\right)=0$ by Proposition 4.8 and so $H_{1}\left(\mathscr{C}_{1}^{0}\right) \cong H_{1}\left(\mathscr{C} \mathscr{K}_{1}^{0}\right)$ by (4.11.1). Thus the "error term" in (6.1) is given by the corank of the linear transformation

$$
\bigoplus_{\sigma \in \Delta_{2}} P_{1} / P_{0} \stackrel{\delta_{1}^{0}}{\longrightarrow} \bigoplus_{e \in \Delta_{1}^{\circ}} P_{1} /\left(P_{0}+\left(P_{1} \cap I_{e}\right)\right)
$$

(compare with (5.4)).

As in the last section (assuming distinct vertex coordinates), the matrix of $\delta_{1}^{0}$ is equivalent to the matrix $\bar{D}$ obtained from the vertex-edge incidence matrix of $G(\Delta)$ by substitution of \pm 1 or 0 times the $2 \times 1$ blocks

$$
\left[\begin{array}{l}
x_{i}-x_{j} \\
y_{i}-y_{j}
\end{array}\right]
$$


for each entry. Thus we have

PROPOSITION 6.2. For any polygonal decomposition $\Delta$ of a 2-disk in $\mathbf{R}^{2}$,

$$
\operatorname{dim} S_{1}^{0}(\Delta)=3 f_{2}-2 f_{1}^{\circ}+f_{0}^{\circ}+\operatorname{corank}(\bar{D}),
$$

where corank $(\bar{D})$ is the number of columns of $\bar{D}$ minus its rank. Further, if corank $(\bar{D})$ $=0$ then

for all $m \geq 0$.

$$
\operatorname{dim} S_{m}^{0}(\Delta)=\left(\begin{array}{c}
m+2 \\
2
\end{array}\right) f_{2}-(m+1) f_{1}^{\circ}+\hat{f}_{0}^{\circ}
$$

PROOF. The last statement follows from Lemma 5.1 and the Euler equation for $\Delta$ (in the case $m=0$ ).

When $\Delta$ is a simplicial 2-disk, $\operatorname{corank}(\bar{D})=0$ as long as none of the triangles degenerates to a line. This follows directly since in the simplicial case, $\operatorname{dim} S_{1}^{0}(\Delta)=$ $f_{0}^{\circ}$ and $3 f_{2}=2 f_{1}^{\circ}$. However for nonsimplicial disks, the corank of $\bar{D}$ will not vanish for certain embeddings, showing that $S_{1}^{0}(\Delta)$ depends upon geometric as well as combinatorial factors, as does $S_{2}^{1}(\Delta)$ in the simplicial case. See [29] for examples.

The "error term" in (4.4) for $r>1$ can be approached in the same way. For a 2-disk $\Delta$, the dimension of $H_{1}\left(\mathscr{C}_{r+1}^{r}\right)$ can be calcualted as the corank of a matrix obtained by substitution of an $(r+2) \times(r+1)$ block in the vertex-edge incidence matrix of $G(\Delta)$. Here, even in the simplicial case, one cannot expect $H_{1}\left(\mathscr{C}_{r+1}^{r}\right)$ to vanish generically. In particular $H_{1}\left(\mathscr{C}_{3}^{2}\right)$ will not vanish if $\Delta$ has an interior vertex of degree 3; this follows, for example, from Schumaker's lower bound [19].

Another problem of some interest is to study piecewise polynomial functions which vanish on the boundary of $\Delta$ with the appropriate degree of smoothness. This is easily incorporated by changing the complex (3.1) by substituting $\Delta_{i}$ for $\Delta_{i}^{\circ}, i=0, \ldots, d-1$. This is essentially switching to usual homology from homology relative to the boundary. The calculation of the Euler characteristic in Theorem 3.3 remains unchanged except for the substitution $f_{i}$ for $f_{i}^{\circ}$. The first real change is that one no longer should expect $H_{0}$ to vanish, although its calculation should be straightforward. The rest of the analysis for planar manifolds should not be all that difficult.

A variation is to ask for different degrees of smoothness along different faces of dimension $d-1$. This can be accomplished by varying the exponents on the ideals $I_{\tau}$ in (3.1), although some care must be taken on the exponents on the rest of the $I_{\lambda}, \ldots, I_{v}$ in $(3.1)$ in order for it to remain a complex (i.e., $\left.\partial \partial=0\right)$. It seems that assigning to each face the maximum $r$ of any $d-1$ face containing it should work.

One possibility to vary the technique of $\S 3$ is to use something other than the space of functions $P_{m}$ in (3.1). This idea has been applied to the study of divergence-free $d$-tuples of piecewise polynomials over $\Delta$, yielding, in dimension 2 , a relationship between the dimensions of such spaces and the dimensions of spaces of ordinary piecewise polynomials [15].

\section{REFERENCES}

1. P. Alfeld, On the dimension of multivariate piecewise polynomial functions, Proc. Biennial Dundee Conf. on Numerical Analysis, Pitman, London, 1985.

2. _ New Trends (G. E. Farin, ed.), SIAM, Philadelphia, Pa.. 1987.

3. P. Alfeld, B. Piper and L. L. Schumaker, An explicit basis for $C^{1}$ quartic bivariate splines, SIAM J. Numer. Anal. 24 (1987), 891-911. 
4. __ Minimally supported bases for spaces of bivariate piecewise polynomials of smoothness $r$ and degree $d \geq 4 r+1$, Comput. Aided Geom. Des. 4 (1987), 105-123.

5. __ Spaces of bivariate splines on triangulations with holes, J. Approx. Theory Appl. (to appear).

6. P. Alfeld and L. L. Schumaker, The dimension of bivariate spline spaces of smoothness $r$ for degree $d \geq 4 r+1$, Const. Approx. 3 (1987), 189-197.

7. R. H. Bartels, Splines in interactive computer graphics, Numerical Analysis (D. F. Griffiths, ed.), Lecture Notes in Math., vol. 1066, Springer-Verlag, New York, 1984, pp. 1-29.

8. L. J. Billera, The algebra of continuous piecewise polynomials over a simplicial complex, Adv. in Math. (to appear).

9. C. K. Chui and R. H. Wang, On smooth multivariate spline functions, Math. Comp. 41 (1983), 131-142.

10. __ Multivariate spline spaces, J. Math. Anal. Appl. 94 (1983), 197-221.

11. P. Ciarlet, Lectures on the finite element method, Tata Institute of Fundamental Research, Bombay, 1975.

12. R. Courant, Variational methods for the solution of problems of equilibrium and vibration, Bull. Amer. Math. Soc. 49 (1943), 1-23.

13. H. Crapo and J. Ryan, Spatial realizations of linear scenes, Structural Topology 13 (1986), 33-68.

14. W. Dahmen and C. A. Micchelli, Recent progress in multivariate splines, Approximation Theory IV (C. K. Chui, L. L. Schumaker and J. D. Ward, eds.), Academic Press, New York, 1983, pp. 27-122.

15. R. Haas, Dimension and bases for certain classes of splines: a combinatorial and homological approach, Ph.D. thesis, Cornell Univ., August 1987.

16. J. R. Munkres, Elements of algebraic topology, Addison-Wesley, Menlo Park, Calif., 1984.

17. J. Morgan and R. Scott, $A$ nodal basis for $C^{1}$ piecewise polynomials of degree $n \geq 5$, Math. Comp. 29 (1975), 736-740.

18. The dimension of the space of $C^{1}$ piecewise polynomials, unpublished manuscript, 1975.

19. L. L. Schumaker, On the dimension of spaces of piecewise polynomials in two variables, Multivariate Approximation Theory (W. Schemp and K. Zeller, eds.), Birkhäuser, Basel, 1979, pp. 396-412.

20. $\ldots$, Bounds on the dimension of spaces of multivariate piecewise polynomials, Rocky Mountain J. Math. 14 (1984), 251-264.

21. E. Spanier, Algebraic topology, Springer-Verlag, New York, 1982.

22. P. F. Stiller, Certain reflexive sheaves on $P_{C}^{n}$ and a problem in approximation theory, Trans. Amer. Math. Soc. 279 (1983), 125-142.

23. G. Strang, Piecewise polynomials and the finite element method, Bull. Amer. Math. Soc. 79 (1973), 1128-1137.

24. _ The dimension of piecewise polynomial spaces and one-sided approximation, Proc. Conf. Numerical Solution of Differential Equations (Dundee 1973), Lecture Notes in Math., vol. 365, Springer-Verlag, New York, 1974, pp. 144-152.

25. R. H. Wang, The structural characterization and interpolation for multivariate splines, Acta. Math. Sinica (Shu Hsueh Hsueh Pao) 18 (1975), 91-106; English transl., 18 (1975), 10-39.

26. N. White and W. Whiteley, The algebraic geometry of stresses in frameworks, SIAM J. Algebraic Discrete Methods 4 (1983), 481-511.

27. W. Whiteley, A matroid on hypergraphs, with applications in scene analysis and geometry, preprint, Champlain Regional College, March 1986.

28. __ A matrix for splines, J. Approx. Theory (to appear).

29. __ The analogy between multivariate splines and hinged panel structures, preprint, Champlain Regional College, June 1986.

30. O. Zariski and P. Samuel, Commutative algebra, Vol. I, Van Nostrand, Princeton, N.J.; Springer-Verlag, New York, 1958.

Department of Mathematics, Cornell University, IthaCA, NeW York 14853

Department of Mathematics, Rutgers University, New Brunswick, New JerSEY 08903 